\title{
Short-lived and nonphosphorescent triplet state of Mexoryl SX, a UV-A sunscreen
}

\author{
Yuta Shamoto ${ }^{1}$, Ryohei Shimizu ${ }^{1}$, Mikio Yagi ${ }^{* 1}$, Nozomi Oguchi-Fujiyama ${ }^{2}$, Jasmin Kang ${ }^{2}$, Azusa \\ Kikuchi *1 \\ ${ }^{1}$ Department of Chemistry, Graduate School of Engineering Science, Yokohama National University, \\ Tokiwadai, Hodogaya-ku, Yokohama 240-8501, Japan. \\ ${ }^{2}$ Shiseido Global Innovation Center, Takashima, Nishi-ku, Yokohama 220-0011, Japan.
}

\begin{abstract}
Mexoryl SX (terephthalylidene-3,3'-dicamphor-10,10'-disulfonic acid, Ecamsule) is a watersoluble UV-A absorber. The near-IR phosphorescence spectrum of singlet oxygen generated by photosensitization with Mexoryl SX was not observed in air-saturated water. On the other hand, the time-resolved near-IR phosphorescence spectrum was observed in oxygen-saturated phosphate buffer ( $\mathrm{pH}$ 7.4). The quantum yield of the singlet oxygen generation $\left(\Phi_{\Delta}\right)$ was determined to be $0.0021 \pm 0.0005$. The ability of Mexoryl SX as a photosensitizer is quite low. The question arises as to the quite low $\Phi_{\Delta}$ value. No phosphorescence was detectable from Mexoryl SX in ethanol at $77 \mathrm{~K}$. We elucidated the nature of the lowest excited triplet $\left(\mathrm{T}_{1}\right)$ state of Mexoryl SX using a time-resolved EPR technique because this technique is powerful for the study of short-lived and non-phosphorescent $T_{1}$ molecules. The strong time-resolved EPR signals were observed. This fact shows that a considerable proportion of the lowest excited singlet $\left(\mathrm{S}_{1}\right)$ state molecules undergoes intersystem crossing (ISC) to the $T_{1}$ state and the deactivation process of the $T_{1}$ state is mainly radiationless. The observed zero-filed splitting parameters, $T_{1}$ lifetime and $S_{1} \rightarrow T_{1}$ ISC anisotropy suggest that the $T_{1}$ state can be regarded as a ${ }^{3} n \pi^{*}{ }^{3} \pi \pi^{*}$ mixing state in character and the two unpaired electrons in the $T_{1}$ state do not localize on (4methylbenzylidene)camphor, a closely related component. Although the shorter $\mathrm{T}_{1}$ lifetime (47 ns) prevents $T_{1}$ state quenching by ground-state oxygen, the ${ }^{3} n \pi^{*}$ character may contribute something to the low $\Phi_{\Delta}$ value.
\end{abstract}

Corresponding authors.

E-mail addresses: yagi-mikio-sc@ynu.ac.jp (M. Yagi), kikuchi-azusa-rh@ynu.ac.jp (A. Kikuchi)

\section{Introduction}


Solar UV radiation exhibits a number of harmful effects on human skin and eye. Numerous scientists the world over have been indicating a high correlation between solar UV exposure and skin cancer, premature photoaging, photodermatoses and accumulation of wrinkles [1-4]. They have also been suggesting a strong correlation between UV exposure and the development of cataract, keratopathy and pterygium [5]. Excessive exposure to solar UV radiation has resulted in an increasing demand to protect human skin and eye against the detrimental effects of solar UV radiation [5-7].

Sunscreen should protect not only against UV-B $(280-320 \mathrm{~nm})$ radiation, but also against the harmful effects of the more deeply penetrating UV-A (320-400 nm) radiation. UV-A radiation indirectly damage DNA of keratinocytes via free radical-mediated pathway [6,7]. UV-A is absorbed by endogenous photosensitizers, such as porphyrins, riboflavin and heme, which induces the generation of reactive oxygen species [6].

Mexoryl SX (terephthalylidene-3,3'-dicamphor-10,10'-disulfonic acid, Ecamsule, Scheme 1) is a watersoluble UV-A absorber [8]. This UV-A absorber has been a proprietary absorber from L'Oréal. Mexoryl SX is a (4-methylbenzylidene)camphor (4-MBC, Scheme 1) sulfonic acid derivative [9]. Mexoryl SX shows excellent photostability under solar UV irradiation. Although it is not approved in the USA, it is widely used in sunscreen products (up to 10\%) in Australia, ASEAN, Canada, EU, Japan and MERCOSUR [10].

A sunscreen molecule absorbs UV radiation and is photoexcited to a higher energy state which is a potentially reactive state. An ideal sunscreen molecule is expected to have the ability to dissipate the absorbed UV energy rapidly and relax back to the ground state without forming any harmful species. However, very little is known about the excited states of Mexoryl SX. To the best of our knowledge only one study was reported by Cantrell et al. in scientific literature [11]. They reported the results of a nanosecond laser flash photolysis investigation of Mexoryl SX.

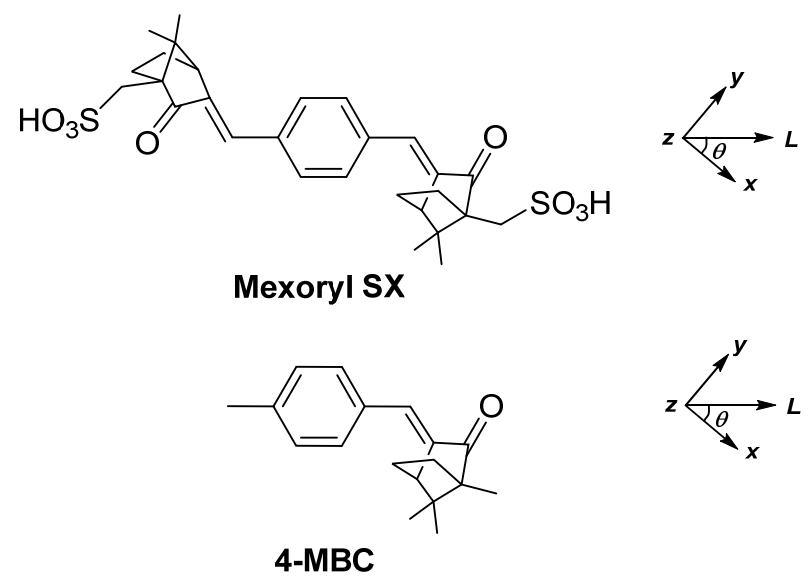

Scheme 1. Molecular structures and principal axes $(x, y, z)$ of the ZFS tensor chosen for Mexory SX and 4-MBC. 
In this paper we report the results of time-resolved near-IR phosphorescence studies of Mexory SXphotosensitized singlet oxygen generation in phosphate buffer $(\mathrm{pH} \mathrm{7.4)}$ at room temperature and timeresolved electron paramagnetic resonance (EPR) studies of the lowest excited triplet $\left(\mathrm{T}_{1}\right)$ state of Mexoryl SX.

\section{Experimental}

\subsection{Chemicals}

Phenalenone was obtained from Aldrich and used as received. Ethanol (Super Special Grade) and phosphate buffer solution ( $\left.0.1 \mathrm{~mol} \mathrm{dm}^{-3}, \mathrm{pH} 7.4\right)$ were obtained from FUJIFILM Wako and used as received. Mexoryl SX was donated by Shiseido Co., Ltd.

\subsection{Optical measurements}

The UV absorption spectra were measured with a JASCO V-550 spectrometer. The fluorescence spectra were measured with a JASCO FP-6500 spectrofluorometer. For the phosphorescence measurements, the samples were excited with an Asahi Spectra REX-250 Hg lamp. The emissions from the samples were passed through a Horiba Jobin Yvon H-20VIS spectrometer and detected by a Hamamatsu Photonics R374 photomultiplier tube.

The details of the time-resolved near-IR phosphorescence measurements are the same as those reported previously [12]. Sample solutions were excited with a Continuum Surelite Nd:YAG laser (355 nm). The emissions were passed through an Asahi Technoglass IR-D80A glass filter (transmits the wavelength 800$3000 \mathrm{~nm}$ ) and a Shimazdu SPG-120IR monochromator and then detected by a Hamamatsu Photonics H10330A-45 near-IR photomultiplier tube module equipped with a Hamamatsu Photonics C999 amplifier unit (10 MHz). The output signal was fed into a Tektronix TDS 3012C digital phosphor oscilloscope (100 MHz) and a Stanford Research SR250 boxcar integrator equipped with a Keyence NR-2000 data acquisition system.

\subsection{EPR measurements}

The experimental setup for the EPR measurements is essentially the same as that reported previously $[13,14]$. The steady-state EPR measurements were carried out using a JEOL-JES-FE1XG X-band spectrometer. A Canrad-Hanovia Xe-Hg lamp equipped with an Asahi Technoglass UV-D33S glass filter (transmits the wavelength $250-400 \mathrm{~nm}$ ), $5 \mathrm{~cm}$ of water filter and a Copal DC-494 electromechanical shutter was used as an excitation source. The external magnetic field was calibrated with an Echo Electronics EFM2000 proton NMR gauss meter. The output signal was fed into an Electronica ELK-5125 waveform storage and a personal computer. For the lifetime measurements, the signals were averaged 512 traces to improve the $\mathrm{S} / \mathrm{N}$ ratio. 
The time-resolved EPR measurements were carried out using a JEOL-JES-FA200 X-band spectrometer without magnetic field modulation. The external magnetic field was calibrated with an Echo Electronics EFM-2000AX proton NMR gauss meter. The samples were excited with the Continuum Surelite Nd:YAG laser $(355 \mathrm{~nm}$, repetition rate $10 \mathrm{~Hz}$ ). For the magnetophotoselection experiments, a Glan-Thompson polarizer was placed between the laser and the sample to polarize the laser light. The output of the diode of the EPR spectrometer was fed into a Tektronix TDS 3032B digital phosphor oscilloscope (300 MHz) and the Stanford Research SR250 boxcar integrator.

\section{Results and discussion}

\subsection{UV absorption and Emission spectra}

The UV absorption spectrum of Mexoryl SX in phosphate buffer (pH 7.4), shown in Fig. 1, is similar to that observed in water by Cantrell et al. [11]. The absorption maximum was observed at $345 \mathrm{~nm}$. DFT calculations were carried out to analyse the UV absorption spectrum using Spartan'18 version 1.2.0 (Wavefunction Inc., CA, USA). Fig. 2 shows the frontier molecular orbitals calculated by Spartan'18 at the B3LYP/6-31G* level in vacuum. The principal excitation leading to absorption in the UV-A region of the spectrum was characterized as being a HOMO-LUMO transition.

No fluorescence was detectable from Mexoryl SX in ethanol at 77 K. Cantrell et al. reported that Mexoryl SX is nonfluorescent in ethanol or water, but weakly fluorescent in acetonitrile at room temperature [11]. The lowest excited singlet $\left(\mathrm{S}_{1}\right)$ state is nonfluorescent or very weakly fluorescent in ethanol. We tried to observe the phosphorescence spectrum of Mexoryl SX in ethanol at $77 \mathrm{~K}$ using a phosphoroscope with a 2 ms dead time. However, no phosphorescence was detectable from Mexoryl SX. As is mentioned in the

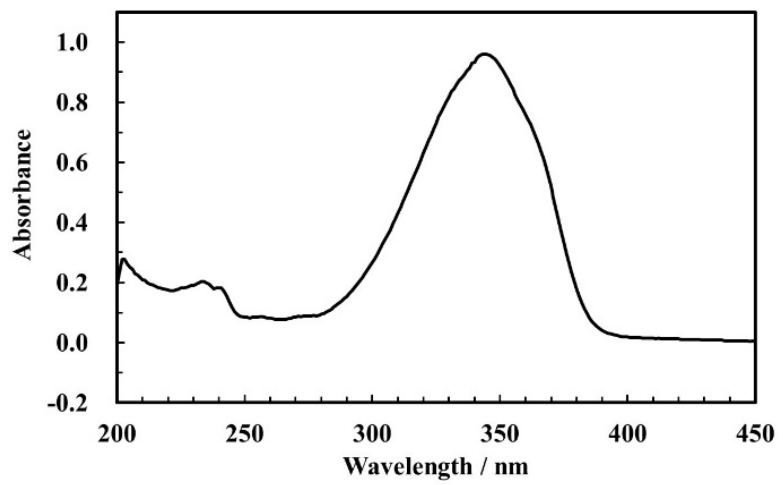

Fig. 1. (a) UV absorption spectrum of Mexoryl SX in phosphate buffer (pH 7.4) at $25^{\circ} \mathrm{C}$. 


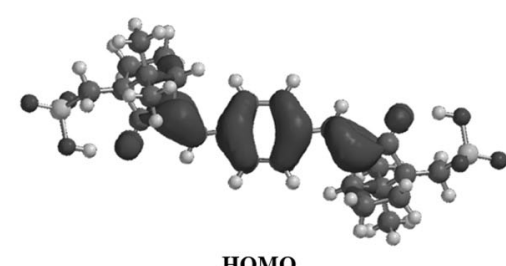

HОМо

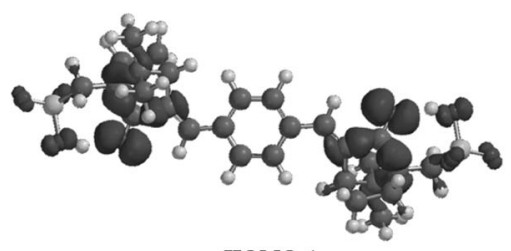

HOMO-1

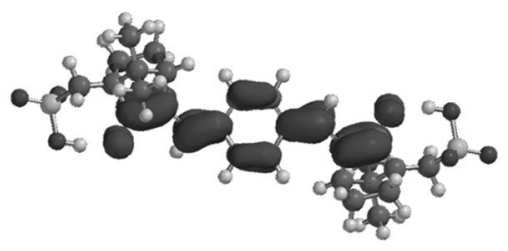

LUMO

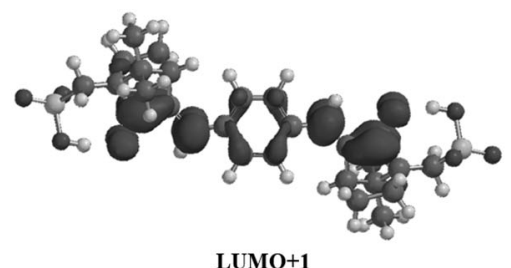

LUMO+1

Fig. 2. Frontier molecular orbitals of Mexoryl SX obtained by DFT calculations. Calculated by Spartan'18 at the B3LYP/6-31G* level in vacuum.

following section, the lifetime of the $\mathrm{T}_{1}$ state of Mexoryl SX is shorter than $10 \mathrm{~ms}$ (longer than $2 \mathrm{~ms}$ ) in ethanol at $77 \mathrm{~K}$. The $\mathrm{T}_{1}$ state is nonphosphorescent or very weakly phosphorescent.

\subsection{Near-IR phosphorescence of singlet oxygen}

It is well known that the most important method of generating singlet oxygen, ${ }^{1} \mathrm{O}_{2}\left({ }^{1} \Delta_{\mathrm{g}}\right)$, in solution is quenching of the $\mathrm{T}_{1}$ states of organic molecules by ground-state oxygen, ${ }^{3} \mathrm{O}_{2}\left({ }^{3} \Sigma_{\mathrm{g}}{ }^{-}\right)$. However, the near-IR phosphorescence spectrum of singlet oxygen generated by photosensitization with Mexoryl SX was too

Table 1

Calculated $\lambda_{\max }$, oscillator strengths and main transitions of Mexoryl SX obtained by TD-DFT calculations ${ }^{\text {a }}$

\begin{tabular}{lll}
\hline$\lambda_{\max }(\mathrm{nm})$ & Oscillator strength & Main transition \\
\hline 366.3 & 1.3406 & HOMO to LUMO $(93 \%)$ \\
353.5 & 0.0004 & HOMO-2 to LUMO $(81 \%)$ \\
353.0 & 0.1244 & HOMO-1 to LUMO $(76 \%)$ \\
303.2 & 0.0111 & HOMO-3 to LUMO $(88 \%)$ \\
\hline
\end{tabular}

${ }^{\text {a }}$ Calculated by Spartan'18 at the B3LYP/6-31G* level in vacuum. 
weak to be observed in air-saturated water at room temperature. We tried to observe the singlet oxygen phosphorescence in oxygen-saturated phosphate buffer ( $\mathrm{pH}$ 7.4). The concentrations of dissolved oxygen in air-saturated and oxygen-saturated water at $25^{\circ} \mathrm{C}$ are 0.27 and $1.27 \mathrm{mmol} \mathrm{dm}^{-3}$, respectively [15]. The wavelength of the singlet oxygen phosphorescence peak (about $1274 \mathrm{~nm}$ ), shown in Fig. 3, is in good agreement with that reported previously in $\mathrm{D}_{2} \mathrm{O}$ by Schmidt (1273.9 nm) [16]. The observed lifetime of singlet oxygen generated by Mexoryl SX-photosensitization, $3.6 \mu \mathrm{s}$, is in good agreement with the previously reported value $3.6 \mu$ s (in air-saturated water) [17].

The use of the nanosecond pulsed laser allows us to analyse the time profile of singlet oxygen phosphorescence after laser shots. The rise and decay times of singlet oxygen phosphorescence, $I_{t}$, are given by the following biexponential function [17-19]:

$$
I_{t}=c \times \Phi_{\Delta}\left\{\tau_{\Delta} /\left(\tau_{\Delta}-\tau_{\mathrm{T}}\right)\right\}\left[\exp \left(-t / \tau_{\Delta}\right)-\exp \left(-t / \tau_{\mathrm{T}}\right)\right]
$$

where $\Phi_{\Delta}$ is a quantum yield of singlet oxygen generation; $\tau_{\Delta}$ and $\tau_{\mathrm{T}}$ are a lifetime of singlet oxygen and a $\mathrm{T}_{1}$ lifetime of a photosensitizer, respectively $\left(\tau_{\mathrm{T}} \neq \tau_{\Delta}\right)$. The proportionality constant $c$ was used to fit Eq. (1) into the actual time profile. The time position $t_{\max }$ of the relative phosphorescence maximum is given as follows:

$$
t_{\max }=\left(\ln \tau_{\Delta}-\ln \tau_{\mathrm{T}}\right) /\left(\tau_{\mathrm{T}}^{-1}-\tau_{\Delta}^{-1}\right) .
$$

We should be careful in estimating the $\Phi_{\Delta}$ values. The phosphorescence maximum depends on $\tau_{\Delta}$ and $\tau_{\mathrm{T}}$ and is not proportional to $\Phi_{\Delta}[20,21]$.

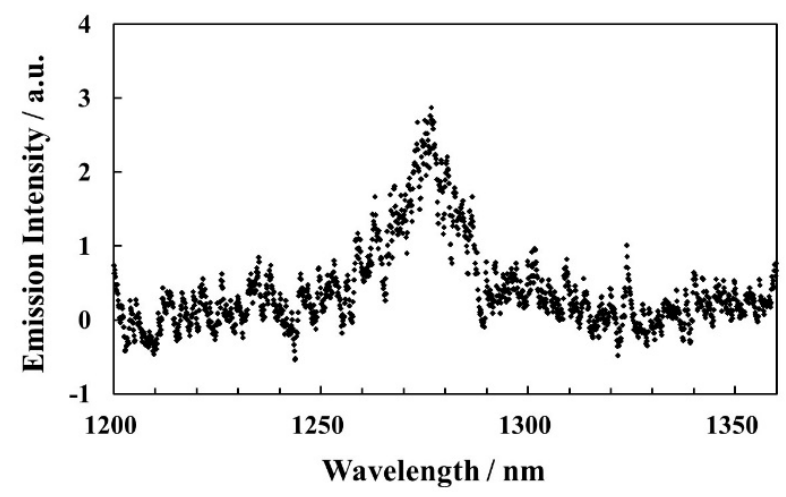

Fig. 3. Time-resolved phosphorescence spectrum of singlet oxygen generated by excitation of Mexoryl SX in oxygen-saturated phosphate buffer $(\mathrm{pH} 7.4)$ at $25^{\circ} \mathrm{C}$. The sampling times were set at $1.5-5.5 \mu$ after the $355 \mathrm{~nm}$ YAG laser pulse. 
Using phenalenone as a reference photosensitizer, the value of $\Phi_{\Delta}$ by Mexoryl SX was determined in oxygen-saturated phosphate buffer ( $\mathrm{pH}$ 7.4). Phenalenone is a water-soluble photosensitizer with $\Phi_{\Delta}$ of $0.98[22,23]$. A sample and a reference should be measured under exactly the same conditions. The absorbance at $355 \mathrm{~nm}$ and energy of the laser pulse for Mexoryl SX were the same as those for phenalenone, 0.84 and $10 \mathrm{~mJ}$, respectively. Fig. 4 shows the time profiles of the phosphorescence intensity of singlet oxygen generated by excitation of phenalenone and Mexoryl SX in oxygen-saturated phosphate buffer $(\mathrm{pH}$ 7.4 ) at $25^{\circ} \mathrm{C}$. In order to observe the initial phosphorescence intensity precisely, the background should be eliminated from the phosphorescence of singlet oxygen. The average of the time profiles at $1200 \mathrm{~nm}$ and $1350 \mathrm{~nm}$ were subtracted from the time profile at $1274 \mathrm{~nm}$, as shown in Figs. S1 and S2 (see Supplementary Material). The results are shown in Fig. 4.
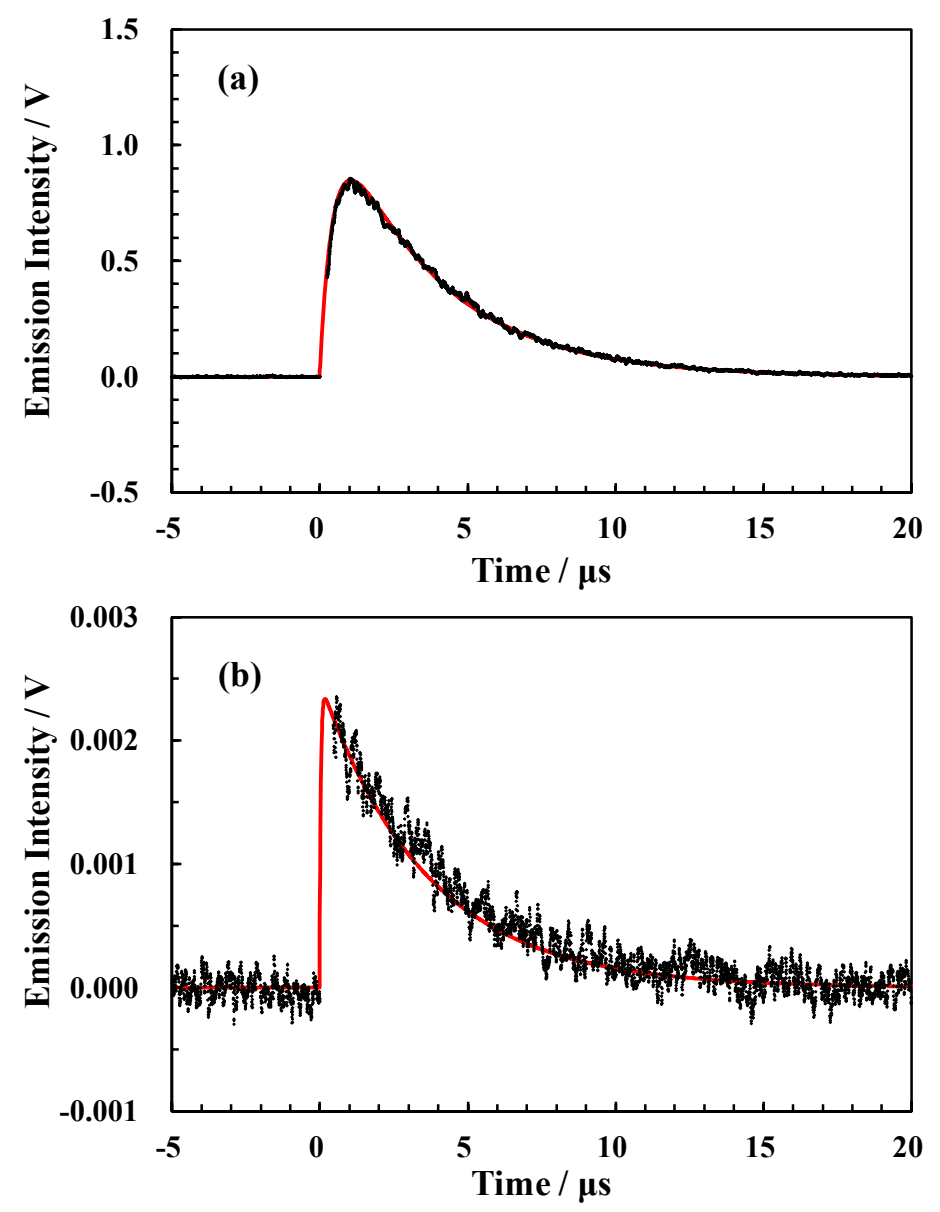

Fig. 4. Time profiles of the phosphorescence intensity of singlet oxygen generated by excitation of (a) phenalenone and (b) Mexoryl SX in oxygen-saturated phosphate buffer ( $\mathrm{pH} 7.4$ ) at $25^{\circ} \mathrm{C}$. Computer-simulated time profiles (solid lines) obtained with (a) $c=1.17 \mathrm{~V}, \Phi_{\Delta}=0.98, \tau_{\Delta}=3.5 \mu$ s and $\tau_{\mathrm{T}}=0.45 \mu \mathrm{s}$, and (b) $c=1.17 \mathrm{~V}, \Phi_{\Delta}=0.0021, \tau_{\Delta}=3.6$ $\mu \mathrm{s}$ and $\tau_{\mathrm{T}}=0.04 \mu \mathrm{s}$. 
The solid lines in Fig. 4 show the time profiles of the phosphorescence intensity calculated using Eq. (1). The $\tau_{\mathrm{T}}$ value of Mexoryl SX was set at $0.04 \mu$ s since $\mathrm{T}_{1}$ lifetime of Mexoryl SX in oxygen-saturated water was determined to be $0.04 \mu$ s at room temperature through the measurements of transient absorption [11]. From the computer-simulated time profiles shown in Fig. 4 (solid lines), the $\Phi_{\Delta}$ value by Mexoryl SX was estimated to be $0.0021 \pm 0.0005$. The ability of Mexoryl SX as a photosensitizer for the singlet oxygen generation is quite low.

\subsection{EPR spectra}

The question arises as to the quite low $\Phi_{\Delta}$ value. We tried to elucidate the nature of the $T_{1}$ state of Mexoryl SX through measurements of EPR spectra since the $T_{1}$ state is nonphosphorescent or very weakly phosphorescent. A time-resolved EPR technique with pulsed laser excitation is a powerful tool for the study of short-lived and very weakly phosphorescent $\mathrm{T}_{1}$ molecules [24]. Ethanol was used as a solvent for EPR and phosphorescence measurements at $77 \mathrm{~K}$ because the cracking frequency at $77 \mathrm{~K}$ is low, $10 \%$ [15]. On the other hand, it is difficult to make water form clear rigid glasses (optically clear frozen solvent) at $77 \mathrm{~K}$.

The EPR spectra of the $\mathrm{T}_{1}$ state in an external magnetic field $\boldsymbol{B}$ are analyzed in terms of the following spin Hamiltonian:

$$
\begin{aligned}
H_{\mathrm{S}} & =g \mu_{\mathrm{B}} \boldsymbol{B} \cdot \boldsymbol{S}+\boldsymbol{S} \cdot \boldsymbol{D} \cdot \boldsymbol{S} \\
& =g \mu_{\mathrm{B}} \boldsymbol{B} \cdot \boldsymbol{S}-X S_{x}{ }^{2}-Y S_{y}{ }^{2}-Z S_{z}{ }^{2} \\
& =g \mu_{\mathrm{B}} \boldsymbol{B} \cdot \boldsymbol{S}+D\left[S_{z}^{2}-(1 / 3) \boldsymbol{S}^{2}\right]+E\left(S_{x}{ }^{2}-S_{y}{ }^{2}\right) .
\end{aligned}
$$

In Eq. (3), $\boldsymbol{D}$ is a fine-structure tensor of principal values of $X, Y$ and $Z$. The relation between the zerofield splitting (ZFS) parameters $(D$ and $E$ ) and the principal values is $D=-3 Z / 2$ and $E=(Y-X) / 2$. The anisotropy of the $g$ tensor is so small that it is often treated as a free electron $g$ value. The principal axes $(x$, $y, z$ ) of the $\boldsymbol{D}$ tensor relative to the molecular axes of Mexoryl SX are not known because the molecular symmetry of Mexoryl SX is poor. The skeleton of Mexoryl SX is carbonyl, ethylenic and phenyl groups. Assuming the molecular structure of the skeleton in the $T_{1}$ state to be planar in rigid ethanol solution at 77 $\mathrm{K}$, the principal axes were taken to be as shown in Scheme 1 . The $x$-axis deviates by an angle of $\theta$ from the $L$-axis. The $L$-axis was taken to be parallel to the single bonds between the phenyl and ethylenic groups.

The steady-state EPR spectrum of the $\mathrm{T}_{1}$ state of Mexoryl SX in ethanol at $77 \mathrm{~K}$ was observed using a Canrad-Hanovia Xe-Hg lamp $(1 \mathrm{~kW})$, as shown in Fig. $5 \mathrm{a}$. The resonance field of the $B_{\min }$ signal gives the value of $D^{*}$ through the following equation [25].

$$
D^{*}=\left\{(3 / 4)(h v)^{2}-3\left(g \mu_{\mathrm{B}} B_{\min }\right)^{2}\right\}^{1 / 2}
$$



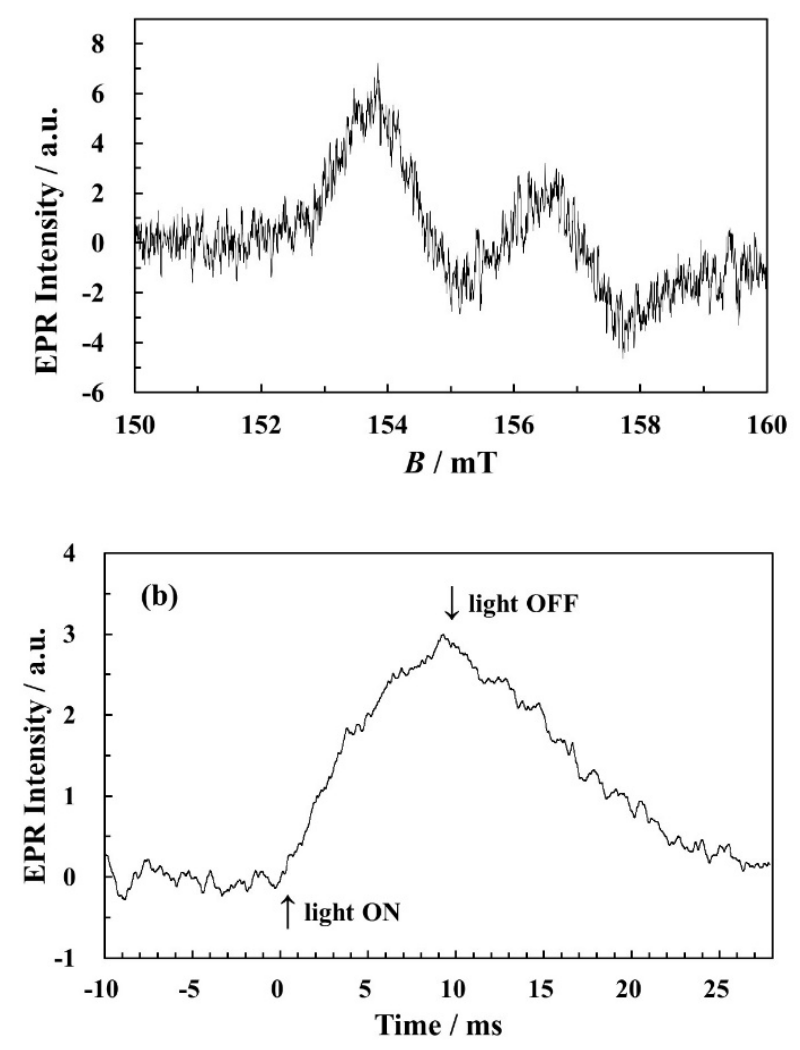

Fig. 5. (a) Steady-state EPR spectrum for the $T_{1}$ state of Mexoryl SX in ethanol at $77 \mathrm{~K}$. (b) Time profile of the steady-state EPR $B_{\min }$ signal at $153.5 \mathrm{mT}$.

where

$$
D^{*}=\left(D^{2}+3 E^{2}\right)^{1 / 2}
$$

$h$ and $v$ have their usual meaning. The $\mathrm{T}_{1}$ lifetime obtained from the decay of the steady-state $B_{\min }$ signal at $153.5 \mathrm{mT}$ is shorter than $10 \mathrm{~ms}$, as shown in Fig. $4 \mathrm{~b}$. The value of $D^{*}$ was estimated to be $0.0926 \mathrm{~cm}^{-1} \mathrm{using}$ Eq. (4). The $\Delta M_{\mathrm{S}}= \pm 1$ transition signals were not observed in the steady-state EPR measurements. This is due to the short lifetime of the $\mathrm{T}_{1}$ state.

On the other hand, the $\Delta M_{\mathrm{S}}= \pm 1$ transition signals are fairly strong in the time-resolved EPR measurements, as shown in Fig. 6b. In general, the intensity of the time-resolved EPR signal does not depend on the $\mathrm{T}_{1}$ lifetime. It depends on the anisotropy in the $\mathrm{S}_{1} \rightarrow \mathrm{T}_{1}$ intersystem crossing (ISC). This is the reason why the EPR $\Delta M_{\mathrm{S}}= \pm 1$ transition signals were observed in the time-resolved measurements. Therefore, the assignment of the observed $\Delta M_{\mathrm{S}}= \pm 1$ transition signals was carried out for the time-resolved spectrum using the magnetophotoselection method. The time-resolved EPR spectra for the $\mathrm{T}_{1}$ state of 
Mexoryl SX obtained with $\boldsymbol{B} \| \boldsymbol{E}$ and $\boldsymbol{B} \perp \boldsymbol{E}$ are shown in Fig. 6d and e. Here $\boldsymbol{E}$ denotes the electric vector of the laser light. Although the direction of the transition moment of Mexoryl SX at $355 \mathrm{~nm}$ has not yet been determined experimentally, we assume that the transition moment is the same as that of trans-cinnamic acid. The polarized UV absorption spectra of trans-cinnamic acid show that in-plane long-axis-polarized absorption takes place dominantly at the longest wavelength band [26]. The in-plane long-axis-polarized absorption at $355 \mathrm{~nm}$ is also suggested by the HOMO-LUMO transition shown in Fig. 2. As is clearly seen in Fig. $6 \mathrm{~d}$ and e, the intensity of $X$ signals is relatively strong with $\boldsymbol{B} \| \boldsymbol{E}$, while the intensity of $Y$ and $Z$ signals is strong with $\boldsymbol{B} \perp \boldsymbol{E}$. As a result, the absolute value of $X$ is larger than those of $Y$ and $Z(|X|>|Z|,|Y|$ and $|D|<3|E|)$.

The problem we are faced with is the signs of $D$ and $E$. The ZFS parameters $D$ and $E$ are calculated from the following equations:

$$
\begin{aligned}
& D=\left(\mu_{0} / 4 \pi\right)(3 / 4)\left(g \mu_{\mathrm{B}}\right)^{2} \int \Phi\left(\boldsymbol{r}_{1}, \boldsymbol{r}_{2}\right)\left[\left(r_{12}{ }^{2}-3 z_{12}{ }^{2}\right) / r_{12}{ }^{5}\right] \Phi\left(\boldsymbol{r}_{1}, \boldsymbol{r}_{2}\right) \mathrm{d} \tau_{1} \mathrm{~d} \tau_{2} \\
& E=\left(\mu_{0} / 4 \pi\right)(3 / 4)\left(g \mu_{\mathrm{B}}\right)^{2} \int \Phi\left(\boldsymbol{r}_{1}, \boldsymbol{r}_{2}\right)\left[\left(y_{12}{ }^{2}-x_{12}{ }^{2}\right) / r_{12}{ }^{5}\right] \Phi\left(\boldsymbol{r}_{1}, \boldsymbol{r}_{2}\right) \mathrm{d} \tau_{1} \mathrm{~d} \tau_{2} .
\end{aligned}
$$

Here, $\Phi\left(\mathbf{r}_{1}, \mathbf{r}_{2}\right)$ is the open shell orbital wavefunction of the $\mathrm{T}_{1}$ state. The $D^{*}$ value and $\mathrm{T}_{1}$ lifetime suggest that the $\mathrm{T}_{1}$ state possesses mainly a ${ }^{3} \pi \pi^{*}$ character. As is known comprehensively, $\mathrm{T}_{z}$ sublevels are the lowest in energy for ${ }^{3} \pi \pi^{*}$ states [27]. Consequently, the order of the $\mathrm{T}_{1}$ sublevels was determined to be $\mathrm{T}_{x}$ $>0>\mathrm{T}_{y}>\mathrm{T}_{z}$ in energy. The signs of $D$ and $E$ are positive and negative, respectively. This is consistent with the signs of $D$ and $E$ expected from the distribution of the two unpaired electrons estimated from the HOMO and LUMO shown in Fig. 2. We can reasonably assign all the observed signals as in Fig. 6.

The $D$ and $E$ values listed in Table 2 were obtained from the six stationary fields of the $\Delta M_{\mathrm{S}}= \pm 1$ transition signals using the following equations [28,29].

$$
\begin{aligned}
& |X|=(1 / 6 h v)\left(g \mu_{\mathrm{B}}\right)^{2}\left(B_{x 2}{ }^{2}-B_{x 1}{ }^{2}\right) \\
& |Y|=(1 / 6 h v)\left(g \mu_{\mathrm{B}}\right)^{2}\left(B_{y 2}{ }^{2}-B_{y 1}{ }^{2}\right) \\
& |Z|=(1 / 6 h v)\left(g \mu_{\mathrm{B}}\right)^{2}\left(B_{z 2}{ }^{2}-B_{z 1}{ }^{2}\right)
\end{aligned}
$$

where $B_{i 1}$ and $B_{i 2}(i=x, y, z)$ are the low and high stationary fields of the $\Delta M_{\mathrm{S}}= \pm 1$ transition signals, respectively. The $D^{*}$ value calculated from the observed $D$ and $E$ values using Eq. (5), $0.0919 \mathrm{~cm}^{-1}$, is in good agreement with that obtained from the steady-state EPR $B_{\min }$ signals using Equation (4), $0.0926 \mathrm{~cm}^{-}$ 1 . 


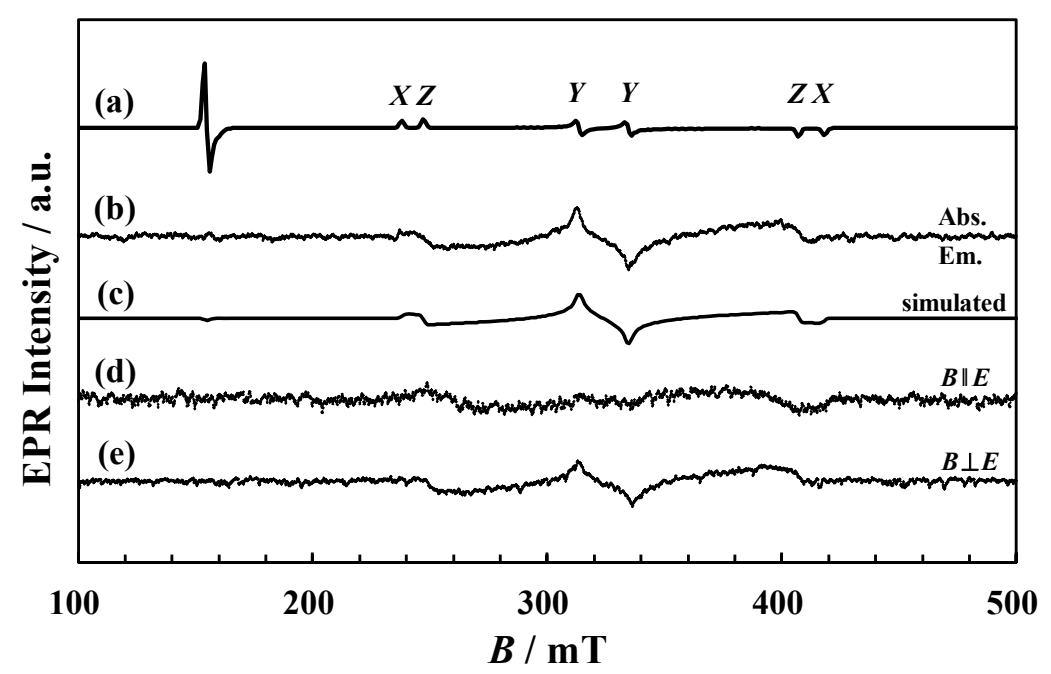

Fig. 6. Computer-simulated (a) steady-state and (c) time-resolved EPR spectra obtained by using $D=0.0743 \mathrm{~cm}^{-}$ ${ }^{1}, E=-0.0312 \mathrm{~cm}^{-1}$ and $\left(P_{x}-P_{z}\right):\left(P_{y}-P_{z}\right)=0.15: 0.85$. Time-resolved EPR spectra for the $\mathrm{T}_{1}$ state of Mexoryl SX in ethanol at $77 \mathrm{~K}$ (b) with nonpolarized light and polarized light (d) with $\boldsymbol{B} \| \boldsymbol{E}$ and (e) with $\boldsymbol{B} \perp \boldsymbol{E}$. The sampling times were set at $0.3-0.8 \mu$ s after the $355 \mathrm{~nm}$ laser pulse.

The time-resolved EPR spectrum shows the AEA/EAE polarization pattern of the $\Delta M_{\mathrm{S}}= \pm 1$ transition signals. A and E denote an absorption and an emission of a microwave, respectively. The observed EPR spectra were simulated with the observed ZFS parameters, microwave frequency of $9.218 \mathrm{GHz}$ and a Gaussian linewidth parameter of $2.0 \mathrm{mT}$. The simulations were carried out using a homemade program (see Supplementary Material). The results are shown in Fig. 6a and c.

Three spin sublevels of the $T_{1}$ state couple with different singlet states via spin-orbit couplings. Consequently, the ISC rate constants to the three spin sublevels are different. The time-resolved EPR spectra were analyzed in terms of the spin Hamiltonian taking account of the relative population ratio in the spin sublevels $\left(P_{x}, P_{y}\right.$ and $\left.P_{z}\right)$. The population ratio was estimated by the computer simulation, as shown in Fig. 6c. The relative populating rates were estimated to be $\left(P_{x}-P_{z}\right):\left(P_{y}-P_{z}\right)=0.15: 0.85$. The $\mathrm{S}_{1} \rightarrow \mathrm{T}_{1}$ ISC of Mexoryl SX is highly anisotropic. We can confirm the facts that the EPR signal of Mexoryl SX with a short $\mathrm{T}_{1}$ lifetime is weak in the steady-state measurements but strong in the time-resolved measurements.

An ordinary steady-state EPR technique is adequate to determine $T_{1}$ lifetimes longer than several milliseconds. One can determine the $T_{1}$ lifetimes from an analysis of the time profile of a time-resolved EPR signal, when the spin-lattice relaxation rates are negligible compared with the $\mathrm{T}_{1}$ decay rates [24]. The spin-lattice relaxation rates are on the order of microseconds at $77 \mathrm{~K}$ [24,31]. The $\mathrm{T}_{1}$ lifetime of Mexoryl SX should be determined through measurements of steady-state EPR signals although the time-resolved EPR signals are much stronger than the steady-state EPR signals.

The steady-state and time-resolved EPR measurements show that a considerable proportion of the $\mathrm{S}_{1}$ state Mexoryl SX molecules undergoes ISC to the $T_{1}$ state, although at present we have no data on the quantum yield of $S_{1} \rightarrow T_{1}$ ISC in ethanol at $77 \mathrm{~K}$. It is impossible to determine absolute quantum yields of 
$\mathrm{S}_{1} \rightarrow \mathrm{T}_{1}$ ISC by means of the time-resolved EPR spectroscopy. Not only the concentration of $\mathrm{T}_{1}$ molecules but also the ZFS values affects the intensity of the steady-state EPR $B_{\min }$ signal [31] The anisotropy in the ISC also affects the intensity of the time-resolved EPR signal [24]. Therefore, it is difficult to determine the quantum yield of $\mathrm{S}_{1} \rightarrow \mathrm{T}_{1}$ ISC through the measurements of EPR spectra.

\subsection{Nature of the lowest excited triplet state}

We tried to discuss the observed short $T_{1}$ lifetime and highly anisotropic triplet sublevel populating rates of Mexoryl SX in terms of mixing between ${ }^{3} n \pi^{*}$ and ${ }^{3} \pi \pi^{*}$ states. The TD-DFT calculations suggest that the HOMO-1 to LUMO transition corresponds to an $n \pi^{*}$ state. In general, the $\mathrm{S}_{1} \rightarrow \mathrm{T}_{1}$ ISC rates to the three $T_{1}$ sublevels are not highly anisotropic for pure ${ }^{3} \pi \pi^{*}$ states of aromatic hydrocarbons [27]. On the other hand, they are highly anisotropic for aromatic carbonyls, where ${ }^{3} \mathrm{n} \pi^{*}$ and ${ }^{3} \pi \pi^{*}$ mixing enhances the anisotropy in the $\mathrm{S}_{1} \rightarrow \mathrm{T}_{1}$ ISC rates to the three $\mathrm{T}_{1}$ sublevels. The ${ }^{3} \mathrm{n} \pi^{*}$ character mixed into the ${ }^{3} \pi \pi^{*}$ state shortens the $T_{1}$ lifetime $[24,32]$.

The $\mathrm{T}_{1}$ sublevel with the large $\mathrm{S}_{1} \rightarrow \mathrm{T}_{1}$ ISC rate is $\mathrm{T}_{z}$ for aromatic carbonyls $(z$ is along the $\mathrm{C}=\mathrm{O}$ direction) [27]. The carbonyl oxygen atom has a large spin-orbit coupling constant and the ${ }^{1,3} n \pi^{*}$ states play an important role in the $\mathrm{S}_{1} \rightarrow \mathrm{T}_{1}$ ISC process. The molecular symmetry of Mexoryl SX is poor and the local symmetry of the carbonyl moiety is important in determining the $\mathrm{S}_{1} \rightarrow \mathrm{T}_{1}$ ISC process. As shown in Scheme 1 , the deviation of the $y$-axis from the $\mathrm{C}=\mathrm{O}$ direction is $\theta-30^{\circ}$. One can expect [33]

$$
P_{x}: P_{y}=\sin ^{2}\left(\theta-30^{\circ}\right): \cos ^{2}\left(\theta-30^{\circ}\right)
$$

In the ${ }^{3} \pi \pi^{*}$ aromatic molecules with planar molecular structures, the out-of-plane sublevel is much less active in the $\mathrm{S}_{1} \rightarrow \mathrm{T}_{1}$ ISC process than in-plane sublevels [27,34]. We expect in a first approximation that $\left(P_{x}-P_{z}\right):\left(P_{y}-P_{z}\right)$ is given by

$$
\left(P_{x}-P_{z}\right):\left(P_{y}-P_{z}\right)=0.15: 0.85 \approx P_{x}: P_{y}=\sin ^{2}\left(\theta-30^{\circ}\right): \cos ^{2}\left(\theta-30^{\circ}\right) .
$$

From Eq. (12) the value $\theta$ is estimated to be $53^{\circ}$. The deviation of the $y$-axis from the $\mathrm{C}=\mathrm{O}$ direction is estimated to be $23^{\circ}$. The principal axis of the ZFS tensor close to the $\mathrm{C}=\mathrm{O}$ direction is $y$ in Mexoryl SX. Therefore, the $T_{1}$ sublevel preferentially populated by $S_{1} \rightarrow T_{1}$ ISC is expected to be $T_{y}$ as observed. The observed $\mathrm{T}_{1}$ lifetime (shorter than $10 \mathrm{~ms}$ ) and anisotropy in the $\mathrm{S}_{1} \rightarrow \mathrm{T}_{1}$ ISC rates are explained in terms of the mixing of the ${ }^{3} n \pi^{*}$ and ${ }^{3} \pi \pi^{*}$ states in the $T_{1}$ state of Mexoryl SX.

The fraction of $T_{1}$ molecules quenched by ground-state oxygen that gives singlet oxygen is denoted by $S_{\Delta}[35,36]$. Many values of $S_{\Delta}$ have been reported [35,36]. In general, the $T_{1}\left(\pi \pi^{*}\right)$ states of polynuclear aromatics are highly efficient at generating singlet oxygen, frequently with $S_{\Delta} \geq 0.8$. For the $\mathrm{T}_{1}\left(\mathrm{n} \pi^{*}\right)$ states of aromatic ketones $S_{\Delta}$ is generally low, $S_{\Delta} \sim 0.3[35,36]$. In Ar-saturated aqueous solution the $\mathrm{T}_{1}$ lifetime 
Table 2

ZFS parameters $\left(D, E, D^{*}, D^{* *}\right), \mathrm{T}_{1}$ lifetimes $(\tau)$, relative populating rates $\left(P_{x}, P_{y}, P_{z}\right)$ and quantum yields of singlet oxygen generation $\left(\Phi_{\Delta}\right)$ in ethanol at $77 \mathrm{~K}$

\begin{tabular}{llllllll}
\hline Molecule & $D\left(\mathrm{~cm}^{-1}\right)$ & $E\left(\mathrm{~cm}^{-1}\right)$ & $D^{* \mathrm{a}}\left(\mathrm{cm}^{-1}\right)$ & $D^{* * \mathrm{~b}}\left(\mathrm{~cm}^{-1}\right)$ & $\tau^{c}(\mathrm{~ms})$ & $\left(P_{x}-P_{z}\right):\left(P_{y}-P_{z}\right)$ & $\Phi_{\Delta}$ \\
\hline Mexoryl SX & 0.0743 & -0.0312 & 0.0919 & 0.0926 & $<10$ & $0.15: 0.85$ & $0.0021 \pm 0.0005^{\mathrm{d}}$ \\
4-MBC $^{\mathrm{e}}$ & 0.0901 & -0.0498 & 0.1247 & 0.124 & 17 & $0.10: 0.90$ & $0.05^{\mathrm{f}}$ \\
\hline
\end{tabular}

${ }^{\mathrm{a}} D^{*}=\left(D^{2}+3 E^{2}\right)^{1 / 2} . \quad{ }^{\mathrm{b}}$ Obtained from the EPR $B_{\min }$ signal. $\quad{ }^{\mathrm{c}}$ Obtained from the decay of the EPR $B_{\min }$ signal. $\quad{ }^{\mathrm{d}}$ In oxygen-saturated phosphate buffer $(\mathrm{pH} 7.4)$ at $25^{\circ} \mathrm{C} . \quad{ }^{\mathrm{e}}$ From ref. $13 . \quad{ }^{\mathrm{f}}$ In air-saturated ethanol at $25{ }^{\circ} \mathrm{C}$.

of Mexoryl SX (47 ns) is shorter than in Ar-saturated acetonitrile (120 ns) [11]. Although the shorter $\mathrm{T}_{1}$ lifetime in aqueous solution prevents $T_{1}$ state quenching by ground-state oxygen, the ${ }^{3} n \pi^{*}$ character may contribute something to the low quantum yield of the singlet oxygen generation by Mexoryl SX.

The lifetime and ZFS parameters of the $\mathrm{T}_{1}$ state of 4-MBC, a closely related molecule, were reported previously and listed in Table 2 . The anisotropy in the $\mathrm{S}_{1} \rightarrow \mathrm{T}_{1}$ ISC rates of 4-MBC is also listed in Table 2 [13]. As can be seen in Table 2, the $D$ and $D^{*}$ values of Mexoryl SX are smaller than those of 4-MBC, suggesting that the two unpaired electrons of Mexoryl SX do not localize on the 4-MBC component in the $\mathrm{T}_{1}$ state. The $\mathrm{T}_{1}$ state of 4-MBC is phosphorescent and its lifetime is $17 \mathrm{~ms}$ in ethanol at $77 \mathrm{~K}$, while the $\mathrm{T}_{1}$ lifetime of nonphosphorescent Mexoryl SX is shorter than $10 \mathrm{~ms}$. The delocalized character of the unpaired electrons of Mexoryl SX may be related to its nonphosphorescent character and short $\mathrm{T}_{1}$ lifetime. On the other hand, the observed anisotropy in the $\mathrm{S}_{1} \rightarrow \mathrm{T}_{1}$ ISC rates of Mexoryl SX is similar to that of 4-MBC. This fact may suggest that the $\mathrm{C}=\mathrm{O}$ direction of one 4-MBC component is parallel to that of another 4MBC component in the $\mathrm{T}_{1}$ state, as shown in Scheme 1.

\section{Conclusions}

The $\Phi_{\Delta}$ value for Mexoryl SX was determined to be $0.0021 \pm 0.0005$ through the measurements of timeresolved near-IR phosphorescence in oxygen-saturated phosphate buffer ( $\mathrm{pH} 7.4)$ at room temperature. The ability of Mexoryl SX as a photosensitizer for singlet oxygen generation is quite low. The results of the EPR studies showed that the $T_{1}$ sate is a ${ }^{3} n \pi^{*}-{ }^{3} \pi \pi^{*}$ mixing state in character. Although the shorter $T_{1}$ lifetime prevents $T_{1}$ state quenching by ground-state oxygen, the mixing of ${ }^{3} n \pi^{*}$ character into the ${ }^{3} \pi \pi^{*}$ state may contribute something to the low quantum yield of the singlet oxygen generation. An ideal UV absorber in cosmetic sunscreens should be photostable and nontoxic without generating singlet oxygen. The elucidation of the $\mathrm{T}_{1}$ state plays an important role in formulating an ideal UV absorber. 


\section{Acknowledgements}

The authors express their thanks to the Instrumental Analysis Center, Yokohama National University, for the use of the EPR spectrometer (JEOL-JES-FA200). This work was supported by JSPS KAKENHI Grant Numbers 24655060 and 19K05877.

\section{References}

1. W. Bäumler, in Singlet Oxygen: Applications in Biosciences and Nanosciences, ed. By S. Nonell, C. Flors, Vol. 2 (The Royal Society of Chemistry, Cambridge, UK, 2016), p. 205.

2.N. A. Shaath, in Sunscreens: Regulations and Commercial Development, ed. By N. A. Shaath (Taylor \& Francis, Boca Raton, USA, 2005), p. 3.

3. B. P. Hibler, S. W. Dusza, S. Q. Wang, in Principles and Practice of Photoprotection, ed. By S. Q. Wang, H. W. Lim (Springer, Cham, Switzerland, 2016), p. 23.

4. S. A. Miller, S. L. Hamilton, U. G. Wester, W. Howard Cyr, Photochem. Photobiol. 68, 63 (1998). 5. M. Shaban, F. Almutawa, in Principles and Practice of Photoprotection, ed. By S. Q. Wang, H. W. Lim (Springer, Cham, Switzerland, 2016), p. 429.

6. A. Fourtanier, D. Moyal, S. Seite, Photochem. Photobiol. Sci. 11, 81 (2012).

7. C. Cole, in Principles and Practice of Photoprotection, ed. By S. Q. Wang, H. W. Lim (Springer, Cham, Switzerland, 2016), p. 275.

8. A. Deflandre, G. Lang, Int. J. Cosmet. Sci. 10, 53 (1988).

9. A. Fourtanier, J. Labat-Robert, P. Kern, C. Berrebi, A. M. Gracia, B. Boyer, Photochem. Photobiol. 55, 549 (1992).

10. S. Daly, H. Ouyang, P. Maitra, in Principles and Practice of Photoprotection, ed. By S. Q. Wang, H. W. Lim (Springer, Cham, Switzerland, 2016), p. 159.

11. A. Cantrell, D. J. McGarvey, L. Mulroy, T. G. Truscott, Photochem. Photobiol. 70, 292 (1999).

12. T. Tsuchiya, A. Kikuchi, N. Oguchi-Fujiyama, K. Miyazawa, M. Yagi, Photochem. Photobiol. Sci. 14, 807 (2015).

13. A. Kikuchi, K. Shibata, R. Kumasaka, M. Yagi, J. Phys. Chem. A 117, 1413 (2013).

14. A. Kikuchi, K. Shibata, R. Kumasaka, M. Yagi, Photochem. Photobiol. Sci. 12, 246 (2013).

15. M. Montalti, A. Credi, L. Prodi, M. T. Gandolfi, Handbook of Photochemistry, 3rd edn. (Taylor \& Francis, Boca Raton, USA 2006).

16. R. Schmidt, J. Phys. Chem. 100, 8049 (1996).

17. S. Nonell, C. Flors, in Singlet Oxygen: Applications in Biosciences and Nanosciences, ed. By S. Nonell, C. Flors, Vol. 2 (The Royal Society of Chemistry, Cambridge, UK, 2016), p. 7. 18. A. A. Krasnovsky Jr., J. Photochem. Photobiol. A 196, 210 (2008). 
19.J. Baier, T. Fuß, C. Pöllmann, C. Wiesmann, K. Pindl, R. Engl, D. Baumer, M. Maier, M. Landthaler, W. Bäumler, J. Photochem. Photobiol. B 87, 163 (2007).

20. R. Shimizu, M. Yagi, A. Kikuchi, J. Photochem. Photobiol. B 191, 116 (2019).

21. S. Fukuchi, M. Yagi, N. Oguchi-Fujiyama, J. Kang, A. Kikuchi, Photochem. Photobiol. Sci. 18, 1556 (2019).

22.D. G. Fresnadillo, S. Lacombe, in Singlet Oxygen: Applications in Biosciences and Nanosciences, ed. By S. Nonell, C. Flors, Vol. 1 (The Royal Society of Chemistry, Cambridge, UK, 2016) p. 105. 23. R. Schmidt, C. Tanielian, R. Dunsbach, C. Wolff, J. Photochem. Photobiol. A 79, 11 (1994).

24. N. Hirota, S. Yamauchi, J. Photochem. Photobiol. C 4, 109 (2003).

25.P. Kottis, R. Lefebvre, J. Chem. Phys. 39, 393 (1963).

26. J. Tanaka, Bull. Chem. Soc. Jpn. 36, 833 (1963).

27. M. Kinoshita, N. Iwasaki, N. Nishi, Appl. Spectrosc. Rev. 17, 1 (1981).

28.P. Kottis, R. Lefebvre, J. Chem. Phys. 41, 379 (1964).

29. M. S. De Groot, J. H. van der Waals, Physica 29, 1128 (1963).

30. R. Furrer, F. Fujara, C. Lange, D. Stehlik, H. M. Vieth, W. Vollmann, Chem. Phys. Lett. 75, 332 (1980).

31.A. Carrington, A. D. McLachlan, Introduction to Magnetic Resonance (Harper \& Row, London, UK 1967).

32. N. Hirota, S. Yamauchi, M. Terazima, Rev. Chem. Intermed. 8, 189 (1987).

33. D. A. Antheunis, B. J. Botter, J. Scmidt, J. H. van der Waals, Mol. Phys. 29, 49 (1975).

34. E. T. Harrigan, N. Hirota, Mol. Phys. 31, 663 (1976).

35. F. Wilkinson, W. P. Helman, A. B. Ross, J. Phys. Chem. Ref. Data 22, 113 (1993).

36.N. J. Turro, V. Ramamurthy, J. C. Scaiano, Modern Molecular Photochemistry of Organic Molecules (University Science Books, Sausalito, USA 2010). 